CLINICAL STUDY

\title{
Prevalence of polycystic ovaries in women with androgenic alopecia
}

Ester Cela, Carole Robertson, Karen Rush, Eleni Kousta ${ }^{1}$, Davinia M White, Helen Wilson, Glenn Lyons ${ }^{3}$, Philip Kingsley ${ }^{3}$, Mark I McCarthy ${ }^{1,2}$ and Stephen Franks

Department of Obstetrics and Gynaecology and ${ }^{1}$ Section of Endocrinology and Metabolic Medicine, Imperial College Faculty of Medicine St Mary's Hospital, London W2 1PG, ${ }^{2}$ Hammersmith Hospital, London W12 ONN, UK and The Philip Kingsley Trichological Clinic, London W1K 6RU, UK

(Correspondence should be addressed to S Franks, Institute of Reproductive and Developmental Biology, Wolfson and Weston Research Centre for Family Health, Imperial College Faculty of Medicine, Hammersmith Hospital, London W12 ONN, UK; Email: s.franks@imperial.ac.uk)

\begin{abstract}
Objective: Although androgenic alopecia is recognised to be a symptom of polycystic ovary syndrome (PCOS), it is not known whether polycystic ovaries (PCO) and associated endocrine abnormalities are present in patients who present with alopecia as a primary complaint. We therefore set out to determine the strength of the association between androgenic alopecia and PCO. We examined the prevalence of ultrasound-based polycystic ovarian morphology and associated clinical and biochemical features in a large multiethnic group of women whose presenting complaint was of alopecia, and in a control group.

Subjects and methods: We studied 89 women of mixed ethnic origin with androgenic alopecia and compared them to 73 control women. A detailed history was taken, anthropometry was performed and assessment of body-hair distribution was made. The presence of PCO was established by pelvic ultrasound scan. Serum gonadotrophins, testosterone, androstenedione, dihydrotestosterone and sex hormone binding globulin concentrations were measured.

Results: Women with alopecia had a higher prevalence of PCO and hirsutism than the control population (PCO: $67 \%$ vs $27 \%, P<0.00001$; hirsutism: $21 \%$ vs $4 \%, P=0.003$ ). Women with alopecia (with or without PCO) had higher testosterone, androstenedione and free androgen index than controls, even though few had frankly abnormal androgens.

Conclusions: These findings confirm an association between androgenic alopecia and PCO, and other symptoms of hyperandrogenaemia. Thus most women who present with androgenic alopecia as their primary complaint also have PCO and have indices of abnormal androgen production. Since PCO is a well known risk factor for development of type 2 diabetes, this association has important implications for long-term management.
\end{abstract}

European Journal of Endocrinology 149 439-442

\section{Introduction}

Androgenic alopecia is one of the most common causes of hair loss in women (1). It is a slowly progressive condition that is associated with significant psychological morbidity in affected women and for which therapeutic options are limited. The diagnosis is subjective; it is based on the exclusion of other causes of hair loss and by the presence of diffuse, diminishing hair diameter, length and density (hairs per square centimetre). Female androgenic alopecia may present in several patterns. Ludwig described the preservation of the frontal hairline with progressive thinning of the crown (2). It may also take a male-pattern form of balding with bitemporal recession (3). With either pattern of hair loss, androgenic alopecia may also be characterised by diffuse reduction in the volume and density of hair.

Polycystic ovary syndrome (PCOS) is the most common endocrine disorder to affect women of reproductive age and is, conventionally, defined as the association of hyperandogenaemia and chronic anovulation in women with polycystic ovaries (PCO) $(4,5)$. Clinical hyperandrogenaemia is represented by hirsutism, acne or alopecia. Alopecia is a recognised feature of PCOS (5-9). PCO occur commonly in women with late onset acne (some studies report a prevalence of between 50 and $75 \%(10,11))$ and in $92 \%$ of women with idiopathic hirsutism (12), but the prevalence of PCO in women who present with alopecia is not 
known. The correlation between clinical and biochemical hyperandrogenaemia is poor, probably due to individual variation in androgen sensitivity of the skin and hair follicles.

Two previous studies have looked at the association between androgenic alopecia and PCOS. Futterweit and colleagues (6) studied 109 women with alopecia. They reported a $28 \%$ prevalence of polycystic ovary 'disease' in their study population; however both their inclusion criteria for the study and their diagnostic criteria for PCOS were different from those used in our study. Specifically, ovarian ultrasound was not routinely used in the diagnosis of PCOS. O'Driscoll and co-workers (8) studied 350 women with 'hirsutism or androgenetic alopecia' (the study does not specify how many women had alopecia and how many had hirsutism) and found a $60 \%$ prevalence of PCO upon ultrasound scan (8). They did not report on androgen levels apart from describing eight patients with clearcut endocrine disorders. To further investigate the association between PCO and androgenic alopecia, we set out to establish the prevalence of PCO morphology and associated clinical and endocrine data in a large multiethnic group of women with a history of androgenic alopecia (who had presented specifically with this symptom to a trichological clinic), and to compare the prevalence in this group with that in a control group of women without alopecia.

\section{Subjects and methods}

\section{Subjects}

Between April 1998 and April 2000, 95 women with androgenic alopecia were referred to the reproductive endocrinology service at St Mary's Hospital, London from a single trichological practice (The Philip Kingsley Clinic, London). All these women had presented initially to the clinic complaining of hair loss. The diagnosis of androgenic alopecia had been made by one of two senior consultant trichologists (GL or PK) based on Ludwig's classification of androgenic alopecia in females (2). Diagnosis was confimed in each case by the second consultant trichologist. The subsequent referral of consecutive patients to St Mary's Hospital was based on the presence of alopecia and none of these women had previously found it necessary to consult a doctor for menstrual disturbances, infertility or hirsutism. None had a known diagnosis of PCOS. A serum ferritin level had been performed in all subjects prior to their referral to St Mary's Hospital; patients with low ferritin were prescribed iron therapy by their GP, and only included in the research project if the treatment made no difference to their alopecia. Thyroid abnormalities were excluded in all participants. Of the 95 women seen, six were excluded from further analysis: three were found to have post-menopausal serum levels of gonadotrophins and three others were
Table 1 Clinical characteristics of women with a primary complaint of alopecia and of the control group of women with no androgenic symptoms.

\begin{tabular}{lccc}
\hline & $\begin{array}{c}\text { Women with } \\
\text { androgenic } \\
\text { alopecia }(n=89)\end{array}$ & $\begin{array}{c}\text { Control women } \\
(n=73)\end{array}$ & $\boldsymbol{P}$ \\
\hline Age (years) & $31.5(27.5-36.0)$ & $36.0(32.5-39.4)$ & $<0.0001$ \\
BMl (kg/m $\left.{ }^{2}\right)$ & $22.3(20.8-25.5)$ & $23.8(21.0-27.5)$ & $\mathrm{NS}$ \\
WHR & $0.78(0.74-0.81)$ & $0.77(0.73-0.81)$ & $\mathrm{NS}$ \\
Menstrual cycle & & & \\
$\quad$ Irregular & $21 / 89(24 \%)$ & $11 / 73(15 \%)$ & $\mathrm{NS}$ \\
$\quad$ Regular & $68 / 89(76 \%)$ & $62 / 73(85 \%)$ & $\mathrm{NS}$ \\
Hirsutism & $19 / 89(21 \%)$ & $3 / 73(4 \%)$ & $<0.003$ \\
Acne & $38 / 89(43 \%)$ & $\mathrm{N} / \mathrm{A}$ & $\mathrm{N} / \mathrm{A}$ \\
\hline
\end{tabular}

Results are expressed as median (interquartile range). BMI, body mass index; WHR, waist-hip ratio; NS, not significant; N/A, not available.

excluded because it was not possible to accurately determine their ovarian morphology. The subjects' characteristics are shown in Table 1.

Over the same period, 73 control women were recruited from St Mary's Hospital antenatal database. These were parous women who did not have a history of alopecia and formed a control cohort for a parallel study of gestational diabetes (13). They were neither pregnant nor breast-feeding at the time of the study. They had not found it necessary to consult a doctor for menstrual disturbance or hirsutism and none had a known diagnosis of PCOS. Data for this group of women have previously been published (13).

The majority of women in both alopecia and control groups were Europid in origin (63/89 with alopecia; $56 / 73$ controls). Of the remaining women, there were more subjects of Middle-Eastern and South Asian origin in the alopecia group $(n=15)$ than in controls $(n=1)$ and fewer of Afro-Carribean origin in alopecia women $(n=2)$ than amongst controls $(n=14)$. All patients gave informed consent for the study which was approved by the ethical committee of St Mary's NHS Trust.

\section{Methods}

All subjects were invited to attend the Metabolic Day Ward at St Mary's Hospital, whilst in the follicular phase of the menstrual cycle when possible. A normal menstrual cycle was defined by a cycle length of between 21 and 35 days with no more than a 7 day variation between cycles in any individual. Medical history was recorded and body mass index (BMI) and waist/hip ratio (WHR) were measured. The presence of androgenic alopecia was confirmed and assessment was made of the presence and degree of hirsutism (defined as a Ferriman-Gallwey score of eight or more). Ovarian morphology was determined by ultrasound scan carried out by one of two experienced ultrasound operators (DMW or HW) and PCO was 
diagnosed according to the criteria established by Adams and colleagues (12).

Biochemical analysis was only performed on women not taking hormonal contraceptives or other medication known to affect the measured parameters. Serum luteinising hormone (LH) and follicle stimulating hormone (FSH) were measured in the follicular phase of the cycle. Testosterone, androstenedione, dihydrotestosterone (DHT) and sex hormone binding globulin (SHBG) concentrations were also measured. Serum LH and FSH were measured by the sandwich immunoassay by Bayer immuno 1 analyser (Bayer Corporation, Tarrytown, NY, USA). Interassay coefficients of variation for FSH and LH were $<2.5 \%$ (14). Testosterone was measured by radioimmunoassay as previously described (12). Androstenedione and DHT were assayed using 'in-house' radioimmunoassays employing ether extraction and dextran coated charcoal separation. ${ }^{3} \mathrm{H}-\mathrm{DHT}$ and androstenedione were obtained from Amersham Biosciences UK Ltd (Chalfont St Giles, UK). The antibody used in the DHT assay was raised in-house whilst the antibody to androstenedione was purchased from Guildhay Ltd (Guildford, UK). As the DHT antibody showed significant cross reaction with testosterone, testosterone was chemically modified to a non-cross-reacting molecule by oxidation to a glycol with potassium permanganate. During the course of this study coefficients of interassay variation for a mid-range value were less than $10 \%$ for androstenedione and less than $15 \%$ for DHT. SHBG was measured using the Immulite method (Euro DPC Ltd, Llanberis, Gwynedd, UK).

\section{Statistical analysis}

Data are presented as medians (25th-75th centiles). Statistical analyses were performed in SPSS 10.0 for Windows using the Mann-Whitney test and $\chi^{2}$ test, as appropriate. As the study population and the control population were not ethnically matched, logistic regression analysis was performed in order to rule out any confounding effect of ethnicity on the prevalence of PCO morphology.

\section{Results}

\section{Comparison between women with androgenic alopecia and control subjects}

Women with androgenic alopecia were younger than the control population but BMI was similar in both groups (Table 1). Of 89 women with a history of alopecia in whom ovarian morphology could be accurately assessed, $60(67 \%)$ had PCO and among the control women $20(27 \%)$ had PCO. The prevalence of PCO was significantly higher in women with alopecia than in the control population $\left(\chi^{2}=24, \quad P<0.00001\right)$ (Table 2). Logistic regression analysis was used to assess the relationship between alopecia and ovarian morphology, whilst allowing for ethnicity-related effects. The presence of alopecia was found to be a major determinant of PCO morphology $(P<0.001$, odds ratio $=6.0)$, whereas ethnicity did not influence ovarian morphology $(P=0.2)$ in this dataset.

In women with alopecia, there was a higher prevalence of hirsutism than in the control population, and $43 \%$ of the study population had acne. The prevalence of menstrual irregularities (24\%) was not significantly different from that in the control group (See Table 1). Only three patients (two with PCO, one without) had both hyperandrogenaemia and irregular menses.

Even though most women had values within the normal range (only four women, three with PCO, had frankly elevated serum testosterone levels, ie. $>3.0 \mathrm{nmol} / \mathrm{l}$ ), women with alopecia had higher mean values of LH, testosterone, androstenedione concentrations and free androgen index than control women (see Table 2). There were no differences between the two groups' FSH, DHT and SHBG concentrations.

Table 2 Ultrasound and biochemical results in patients with a primary complaint of alopecia and in normal controls. Results are expressed as median (interquartile range).

\begin{tabular}{|c|c|c|c|}
\hline & $\begin{array}{l}\text { Women with androgenic } \\
\text { alopecia }(n=89)\end{array}$ & $\begin{array}{l}\text { Control women } \\
\quad(n=73)\end{array}$ & $\boldsymbol{P}$ \\
\hline \multicolumn{4}{|l|}{ Ovarian morphology } \\
\hline PCO & 60/89 (67\%) & 20/73 (27\%) & \\
\hline Normal & $29 / 89$ (33\%) & $53 / 73(73 \%)$ & $<0.00001$ \\
\hline FSH (U/I) & $6.6(5.8-7.5) n=56$ & $6.1(4.6-8.3) n=36$ & NS \\
\hline $\mathrm{LH}(\mathrm{U} / \mathrm{l})$ & $5.6(4.3-7.8) n=56$ & $4.4(3.4-4.9) n=36$ & 0.02 \\
\hline Testosterone $(\mathrm{nmol} / \mathrm{l})$ & $1.7(1.2-2.1) n=65$ & $1.3(0.9-1.6) n=45$ & 0.004 \\
\hline Androstenedione $(\mathrm{nmol} / \mathrm{l})$ & $5.8(4.4-7.1) n=65$ & $4.1(2.8-5.2) n=45$ & $<0.0001$ \\
\hline $\mathrm{DHT}(\mathrm{nmol} / \mathrm{l})$ & $0.8(0.6-1.0) n=31$ & $0.7(0.5-0.8) n=45$ & NS \\
\hline SHBG (nmol/l) & $51(36-67) n=65$ & $59(43-102) n=45$ & NS \\
\hline $\begin{array}{l}\text { Free androgen index } \\
\quad(\text { testosterone/SHBG } \times 100)\end{array}$ & $3.2(2.5-4.9) n=65$ & $1.8(1.2-3.2) n=45$ & 0.01 \\
\hline
\end{tabular}

NS, not significant. 


\section{Discussion}

We found that PCO were very common in a large multiethnic group of women with androgenic alopecia (67\% compared with $27 \%$ in the control group), confirming an association between PCO morphology and alopecia. Cases and controls were matched for BMI; the control group were older but all were premenopausal with normal serum FSH and this is unlikely to have a bearing on the prevalence of $\mathrm{PCO}$ or endocrine features. The majority of the patients with alopecia in this study were of normal weight and there was no difference in BMI between those with and without PCO either in the patient or control populations. This is not surprising, given that patients were recruited on the basis of a primary complaint of alopecia rather than as subjects with classic features of PCOS, amongst whom obesity tends to be more prevalent (5). When looking at the group of women with alopecia as a whole, there was a higher prevalence of hirsutism in this group than in the control population. Testosterone, androstenedione and free androgen index were also higher.

Despite the fact that most values were within the normal range, women with alopecia and PCO had indices of androgen metabolism typical of women with PCOS (ie. elevated median testosterone, androstenedione, free androgen index and lower SHBG).

These results indicate that women with alopecia have a disorder of androgen metabolism, which also manifests clinically in the higher prevalence of hirsutism than in the control population. In most, but not all, cases, alopecia was associated with the presence of PCO. It is interesting to note that androgen dependent symptoms were present even when androgen values were within the normal range. This is likely to be due to an association of increased production and increased clearance of androgens by target tissues, but this was not specifically investigated in this study.

Concentrations of DHT were no higher in women with alopecia than in controls, so measurement of this androgen appears to be no better a marker of androgenicity than total testosterone, androstenedione or free andogen index.

As mentioned in the introduction, the two previous studies that investigated the association between androgenic alopecia and PCOS differed from ours in a number of ways, most significantly in the reason for the primary presentation. In our study, the only reason for these women seeking medical attention was the symptom of alopecia. In summary, we found that PCO occurred in two-thirds of such subjects and many, on further assessment, had other associated symptoms of hyperandrogenaemia, ie. acne and hirsutism. Biochemical indices of androgen excess, consistent with diagnosis of PCOS, were also more common in women with androgenic alopecia than in control subjects. These findings confirm a strong association between androgenic alopecia and PCO. Thus alopecia may be the presenting complaint in women with other features of PCOS including indices of metabolic dysfunction. PCOS is a well known risk factor for development of type 2 diabetes, so this association has important implications for long-term management.

\section{Acknowledgements}

We are grateful to Mandy Donaldson (Endocrine Laboratory, Hammersmith Hospital) for technical assistance with the androgen assays and to Alec Sanderson (HICOM, Surrey, UK) for database design.

\section{References}

1 Stickler JH \& Algin BG. Androgen excess and hair loss in women. In Androgen Excess Disorders, pp 141. Eds R Azzia, JE Nestler \& D Dewailly. New York: Lippincot-Raven, 1997.

2 Ludwig E. Classification of the types of androgenetic alopecia (common baldness) occurring in the female sex. British Journal of Dermatology $197797247-254$.

3 Hamilton JB. Patterned loss of hair in man: types and incidence. Annals of the New York Academy of Sciences 1951 83 708-718.

4 Zawadzki JK \& Dunaif A. Diagnostic criteria for polycystic ovary syndrome: towards a rational approach. In Polycystic Ovary Syndrome, pp 377-384. Eds A Dunaif, JR Givens, FP Haseltine \& GR Merriam. Oxford: Blackwell Scientific Publications, 1992.

5 Franks S. Polycystic ovary syndrome. New England Journal of Medicine $1995333853-861$.

6 Futterweit W, Dunaif A, Yeh HC \& Kingsley P. The prevalence of hyperandrogenism in 109 consecutive female patients with diffuse alopecia. Journal of the American Academy of Dermatology $19885831-836$.

7 Conway GS, Honour JW \& Jacobs HS. Heterogeneity of the polycystic ovary syndrome: clinical, endocrine and ultrasound features in 556 patients. Clinical Endocrinology 198930 459-470.

8 O'Driscoll JB, Mamtora H, Higginson J, Pollock A, Kane J \& Anderson DC. A prospective study of the prevalence of clear-cut endocrine disorders and polycystic ovaries in 350 patients presenting with hirsutism or androgenic alopecia. Clinical Endocrinology $199441231-236$.

9 Green J \& Sinclair R. Skin manifestations of polycystic ovary syndrome. In Polycystic Ovary Syndrome, pp 89. Ed. GT Kovacs. Cambridge: Cambridge University Press, 2000.

10 Betti R, Bencini PL, Lodi A, Urbani CE, Chiarelli G \& Crosti C. Incidence of polycystic ovaries in patients with late-onset or persistent acne: hormonal reports. Dermatologica 1990181 109-111.

11 Eden JA. The polycystic ovary syndrome presenting as resistant acne successfully treated with cyproterone acetate. Medical Journal of Australia 1991155 677-680.

12 Adams J, Polson DW \& Franks S. Prevalence of polycystic ovaries in women with anovulation and idiopathic hirsutism. British Medical Journal 1986293 355-359.

13 Kousta E, White DM, Cela E, McCarthy MI \& Franks S. Polycystic ovaries in women with unexplained infertility. Human Reproduction $1999112720-2723$.

14 Gilling-Smith C, Story H, Rogers V \& Franks S. Evidence for a primary abnormality of thecal cell steroidogenesis in the polycystic ovary syndrome. Clinical Endocrinology 199747 93-99.

Received 18 March 2003

Accepted 30 July 2003 